\title{
Realistic Covariance Generation for the GPM Spacecraft
}

\author{
Siamak G. Hesar ${ }^{1}$ and Matthew Duncan ${ }^{2}$ \\ SpaceNav, Boulder, CO 80301 \\ James $\mathrm{H}$.
NASA Pawloski
3
}

A covariance realism process for NASA's Global Precipitation Measurement (GPM) spacecraft is detailed. The GPM spacecraft is in a low earth orbit, and performs collision avoidance maneuvers few times a year. Currently GPM is below the International Space Station (ISS). So, in addition to cataloged debris objects, GPM must contend with smallsat/cubesat objects that are deployed from the ISS. Both operational scenarios require complete knowledge of the expected GPM prediction errors as a function of time. In this study, we present a method for generating realistic predicted covariance that uses linear propagation of the covariance with the addition of process noise. Further analyses are presented for the process noise "tuning" that generates an inflation factor based on the observed error statistics of the predictive satellite trajectories when compared to the definitive ones. Different tuning strategies are considered and compared via a Goodness-of-Fit testing for the Gaussian properties of the scaled covariance. SpaceNav's realistic covariance generation approach takes into account the contribution of predicted maneuver errors in the increased propagation uncertainty. Corresponding maneuver uncertainty is injected into the state uncertainty, and is used within the collision avoidance process to determine the collision risk for close approach events that follow a maneuver. This is a critical step in the maneuver planning process that provides the satellite operator with an accurate quantification of the collision probability for planned maneuvers. Using this information, an informed decision can be made to proceed with a maneuver if the collision risk is acceptable. This approach is validated by Monte-Carlo simulations and results are presented.

I. Nomenclature

TBD

II. Introduction

NASA's Global Precipitation Measurement (GPM) spacecraft is in a low earth orbit (LEO), and performs collision avoidance maneuvers a few times a year. In particular, GPM spacecraft resides in a 65-degree inclined orbit with a mean geodetic height near $407 \mathrm{~km}$, below the orbit of the

1 Chief Scientist, SpaceNav, Boulder, CO 80301.

2 GPM System Engineer, SpaceNav, Boulder, CO 80301.

${ }^{3}$ GPM Mission Director, NASA Goddard Space Flight Center, Greenbelt, MD 20771. 
International Space Station (ISS)i . So in addition to cataloged debris objects, GPM must contend with different smallsat/cubesat objects that are launched from the ISS. Both operational scenarios require complete knowledge of the expected GPM prediction errors as a function of time. This paper describes an approach for generating realistic predictive covariance for this mission.

Space situational awareness (SSA) requires a comprehensive knowledge of resident space objects (RSO) in near-Earth space environment needed to safeguard and protect space assets from potentially hazardous encounters. An accurate knowledge of the state uncertainties of the orbital objects, including the space assets, is required to perform valid close approach predictions and collision probability assessments. In many SSA applications, the challenge is to represent the proper (realistic) distribution of the states of orbital objects of interest as they are propagated forward in time through pre-defined dynamical systems. It is often noted that the propagated covariance provided by an orbit determination (OD) solution tend to underestimate the true level of dispersion in the predicted trajectories in an operational scenario. Generation of realistic covariance has been used to remedy this issue by simply scaling the covariance bounds by a certain factor at the time of the close approach (TCA). Others have noted that simplifying Gaussian assumption for the distribution of the predicted satellite states may not hold after long propagation durations [1]. More advanced methods have been studied to describe the realistic distribution of RSO's when the Gaussian distribution assumption no longer holds. Gaussian mixture model [2-4], Gauss von Mises model [5], and Polynomial chaos [6] are few examples of such methods. While many of these advanced methods hold promise, they are not widely used operationally. The Joint Space Operations Center (JSpOC) uses the conventional method [7-9] for the computation of collision probability $\left(P_{c}\right)$ at TCA, when it screens the predicted ephemeris files that are provided by the satellite owner/operators ${ }^{\text {ii }}$. This method uses the joint Gaussian distribution of the primary and the secondary objects integrated over a surface containing both objects for the computation of $P_{c}$, and its basic assumption is that both the primary and the secondary states follow a Gaussian distribution. Hence, it is very important to confirm that the predicted covariance generated and delivered to JSpOC for screening is both realistic and also does not violate the Gaussian assumption.

In this study, we present a method that SpaceNav's covariance realism tool (CRT) currently uses for the generation of realistic predicted covariance for the GPM spacecraft. This method involves the propagation of spacecraft uncertainty linearly with the addition of process noise. This has the advantage over the method that simply scales the covariance at the TCA. In that, it produces a covariance matrix that is physically meaningful, i.e. it has been propagated via the orbit dynamical model and can be used to propagate forward and backward in time. Prior to the generation of the realistic covariance, however, we performed a comprehensive overlap comparison analysis of the GPM definitive and predictive ephemeris files to establish a measure of realistic predicted errors for the GPM spacecraft. The result of the overlap analysis is a population of the predicted trajectory errors over the propagation time. A comparison of the observed predicted error profile with the predicted uncertainty profiles provided by the GPM flight operations team (FOT), reveals that the FOT predicted uncertainty underestimates the observed prediction errors, significantly. To correct this, the SpaceNav CRT then uses the propagated covariance matrix inflated by the inclusion of process noise to match with the observed realistic predicted error levels, according to a given metric. This is done by tunning of the process noise parameters via a least squares targeting method until an acceptable convergence is achieved. At each step of the process, a Goodness-of-Fit (GOF) test

\footnotetext{
i https://www.nasa.gov/mission_pages/GPM/spacecraft/index.html

ii https://www.space-track.org/documents/How_the_JSpOC_Calculates_Probability_of_Collision.pdf
} 
is then performed to test for the Gaussian assumption of the predicted error population and the propagated uncertainties. The GOF is also used to test for the performance of different process noise tuning strategies, to identify a metric that best conforms to the Gaussian assumption.

Finally, this paper establishes the validity of SpaceNav's approach for the inclusion of maneuver uncertainty in the predicted covariance, if a predicted maneuver falls within the propagation span. This is done by comparing the implementation of maneuver uncertainty with Monte-Carlo trials reflecting additional uncertainty caused by maneuvers. GPM maneuver error statistics are computed based on the analysis of historical maneuver performance data for this satellite. Corresponding uncertainty is injected into the state uncertainty, and is used within the collision avoidance process to determine the collision risk for close approach events that follow a maneuver. This is a critical step in the maneuver planning process that provides the satellite operator with an accurate quantification of the collision probability for planned maneuvers. Using this information, an informed decision can be made to proceed with a maneuver if the collision risk is acceptable.

\section{An Overview of the GPM Covariance Realism Process}

SpaceNav CRT has been deployed at NASA's Goddard Space Flight Center as part of the GPM flight operations processes, since early 2017. During this time, SpaceNav has provided three major releases of the software to the flight operations team, with the last one delivered on December 01, 2017. It included both manual software package as well as an automated version. There are four main pieces to the CRT; data management utility, overlap analysis, process noise tuning, and realistic covariance generation. The data management utility retrieves the latest ephemeris files and organizes them within the software, and makes sure that the latest space weather and earth orientation parameters are downloaded and updated. The overlap analysis piece performs the overlap comparison analysis between the definitive and the predictive ephemeris files to arrive at a statistically derived measure of the predicted error growth profile. Further details are provided in the following section regarding this process. The computed error growth profile is the target measure of predicted uncertainty that the process noise tunning piece aims to reach in accordance to an specified cost function. Further details on this topic are provided in Section V. Finally, the realistic covariance is generated and written into an orbit ephemeris message (OEM) file by the covariance generation piece. The resultant file, after it passes the required quality assessment checks, is delivered to FOT, which may be used for event screening process by JSpOC and/or collision mitigation maneuver analysis.

Figure 1 shows a high-level process flow of the CRT including the interdependencies between different pieces and inputs and outputs of each section. According to the flow chart, there is a decision making process that occurs outside of the core CRT processes. Its purpose is to involve a human supervision in deciding the final values of the tuned process noise parameters that will be promoted into the operational system. The value of the tuning parameters have a direct impact on the size of the propagated realistic uncertainty bounds. The process noise tuning component of the CRT provides a suggested updated set of tuned parameters to FOT and allows the flight team to approve or reject the promotion of those parameters to the operational level.

A parallel automated CRT process has been running at SpaceNav facility since December 01, 2017. In a normal operational scenario, SpaceNav receives a total of 18 predictive files daily, from GPM FOT that are delivered to a SpaceNav server via a secure file transfer protocol (sftp). FOT delivers three sets of predicted files (6 files in each set) during a day at early morning, mid day, and evening times. Each set of files consists of two types, nominal and no-burn. The nominal file contains a predicted maneuver in it, if the predicted maneuver execution time falls within the 


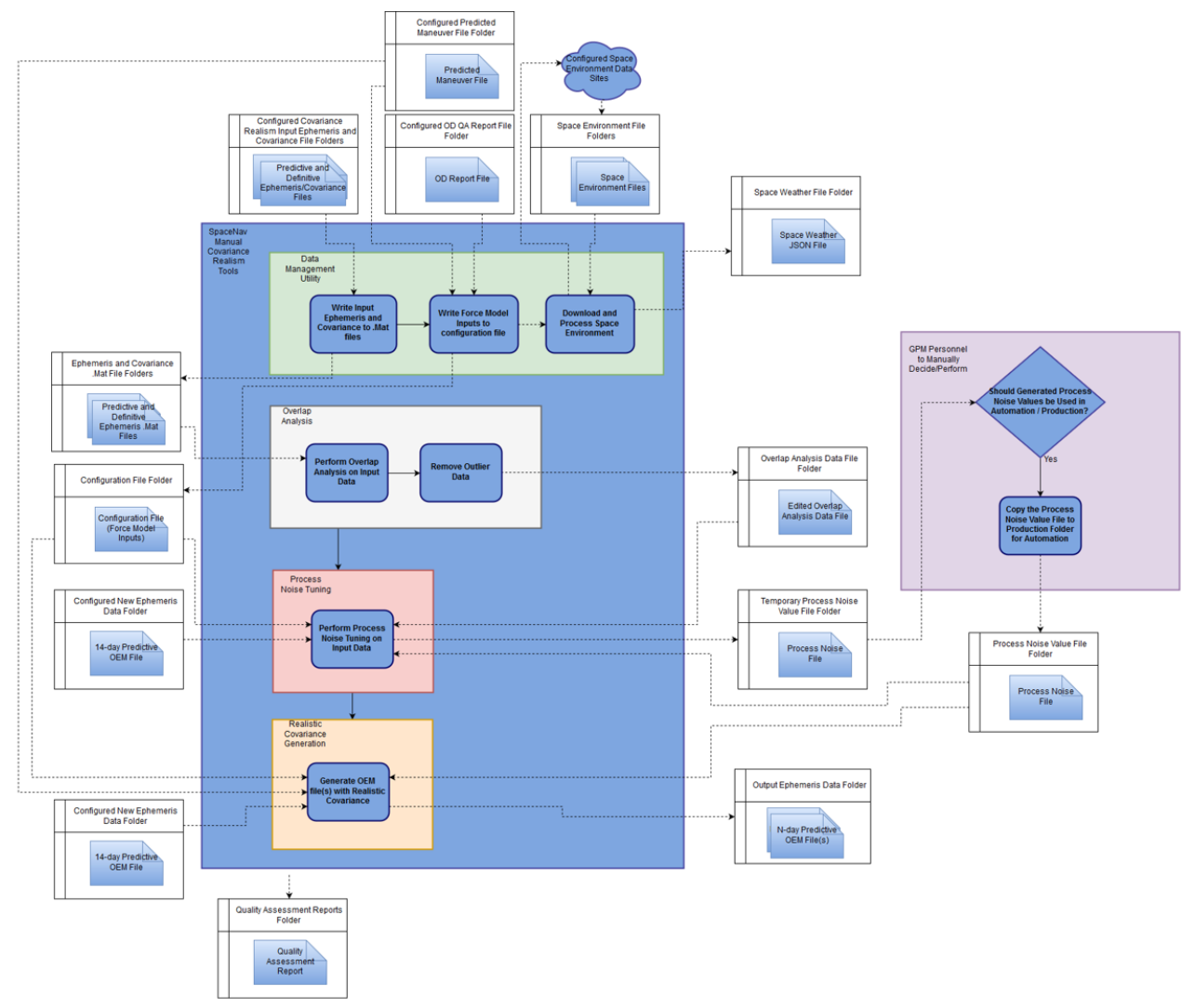

Fig. 1 Functional flow chart of the SpaceNav Covariance Realism Tool.

time span covered by the nominal file. The no-burn file serves as a baseline predictive file that does not contain any maneuvers. Each type includes three different predicted time frames; 7, 9, and 14-day prediction files. At the time of the writing of this paper, a total of 1,528 predicted files and 295 predicted maneuver reports were delivered to SpaceNav and are processed through the automation system starting from December 01, 2017. There exists a quality assessment throughout the entire CRT system and performs a constant evaluation of the steps as well as the generated results and files against predefined checks and benchmarks, and issues error/warning alerts if an issue is detected. The alert messages are generated in accordance to the FOT alert system guidelines and requirements, and can be merged with the operational alert system to provide real-time status of the CRT process to the relevant mission stakeholders.

This gives an high-level overview of the SpaceNav CRT that is currently in place to support the GPM mission. In the following sections, we will dive deeper in some of the key processes within that tool and provide the relevant analyses and results.

\section{Statistical Analysis of the GPM Ephemerides}

\section{A. Overlap comparison analysis}

The covariance realism process starts with an overlap comparison of the "definitive" versus "predictive" ephemeris files generated by the FOT. The overlap analysis gives a measure of the realistic prediction errors and their dispersions. The phrase definitive is used for an ephemeris file that is given by the orbit determination solution. The predictive ephemeris file, on the other hand, is generated by taking the latest orbit determination solution and propagating the satellite trajectory several days into the future. Currently, the GPM FOT generates predicted files up to 
14 days of propagation. Although the data is available for 14 days of propagation, our statistical analysis considers only the first 3 days of propagation. There are two reasons behind this; first, the prediction errors for a LEO satellite increase rapidly (specially down-track) and may start to loose their Gaussian behavior after about 3 to 4 days of propagation [1]; second, often times operational decision making process in regards to an avoidance maneuver occurs within couple of days prior to an estimated close approach event. This is not to say that operations team does not look further than 3 days out into the future for high probability events. Quite the contrary, the mission operations team keeps track of any high probability event that may occur in the future and assesses its evolution over time as the event gets closer, when the prediction accuracy is higher.

For this study, we perform an overlap comparison of the definitive vs. predictive ephemeris files starting from Jan. 01, 2016 up until Mar. 03, 2018, when the analysis was being performed. The overlap comparison process computes the satellite state difference between the predicted states and the corresponding definitive ones that fall within the same time frame. Figure 2 shows a graphical representation of the overlap comparison process. The result of the overlap analysis is a population of the predicted state errors with respect to the prediction time. The dispersion of the predicted error population is a first order measure of the realistic prediction uncertainty. We consider every other 3 predicted file when carrying out the overlap comparison, to make sure that the error population is derived from independent sources.
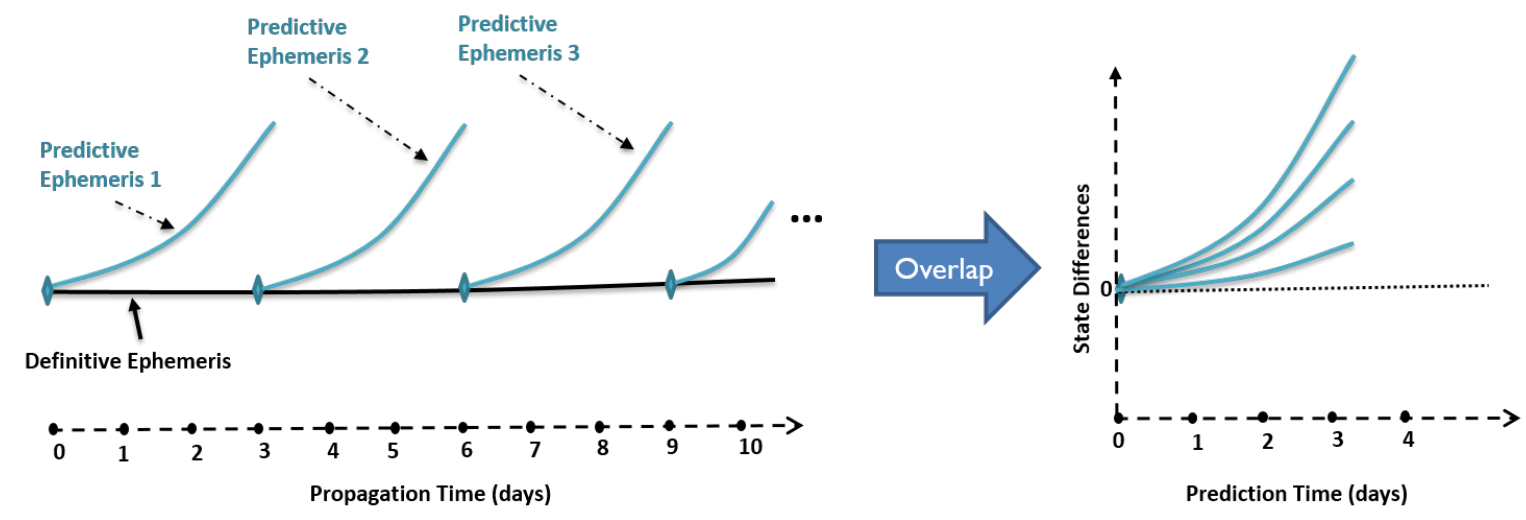

Fig. 2 Schematic view of the overlap comparison process.

Figure 3 shows the prediction errors in the in-track direction of the satellite trajectory as a result of the overlap comparison. This figure shows that there are several cases, where the predicted error increases sharply. This is due to the existence of predicted maneuvers inside those ephemeris files. Errors in the predicted maneuvers (compared to what was actually implemented in the definitive file) result in large errors in the predicted trajectory. In our analysis, we consider those cases as outliers and implement a recursive sigma level procedure to identify and remove those cases. If a predicted file is deemed to be an outlier, the entire file is rejected and not considered in the statistical analysis. The recursive sigma level outlier removal procedure includes two steps. The first step computes the population median and the median absolution deviation (MAD) and rejects the cases whose deviations from the sample median is larger than some multiple (e.g. 10) of the sample MAD. This first iteration is designed to identify and reject the most extreme cases of outliers that would skew the sample mean and standard deviation in the 2nd step, if they were to remain in the population. In the next step, the process continues into a recursive process that identifies the cases, whose deviations from the population mean is larger than some multiple (e.g. 3) of the sample standard deviation and labels them as outliers. This step repeats until no further file is deemed as 
an outlier. After the removal of the outliers from the overlap comparison results, the process is left with a total of 232 files and all of the errors due to the maneuvers are removed, as seen in Figure 4 . This plot shows that the predicted error population show much smoother behavior and they all fit within \pm 3 standard deviation bounds.

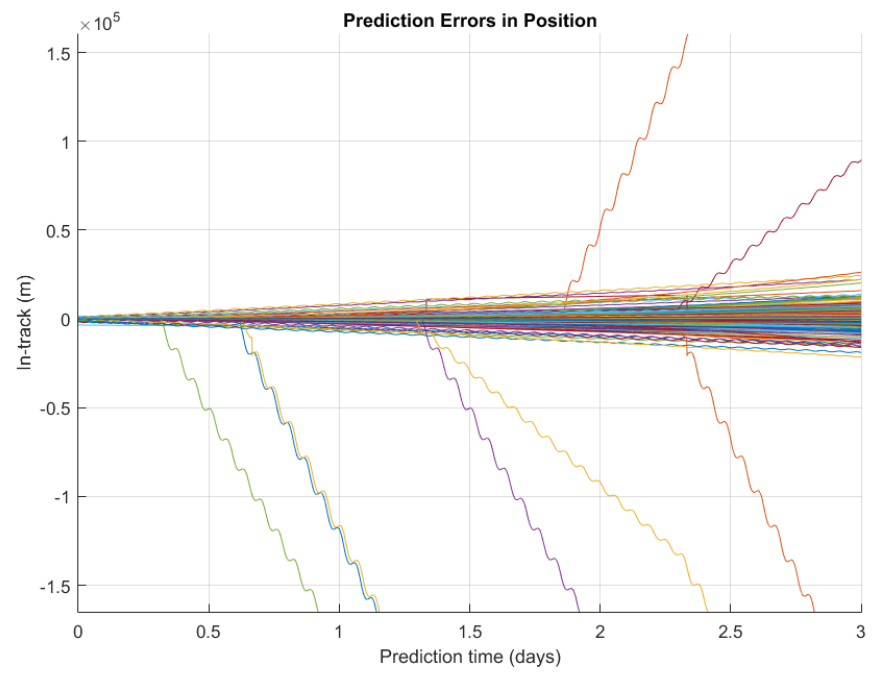

Fig. 3 In-track predicted ephemeris with maneuver errors.
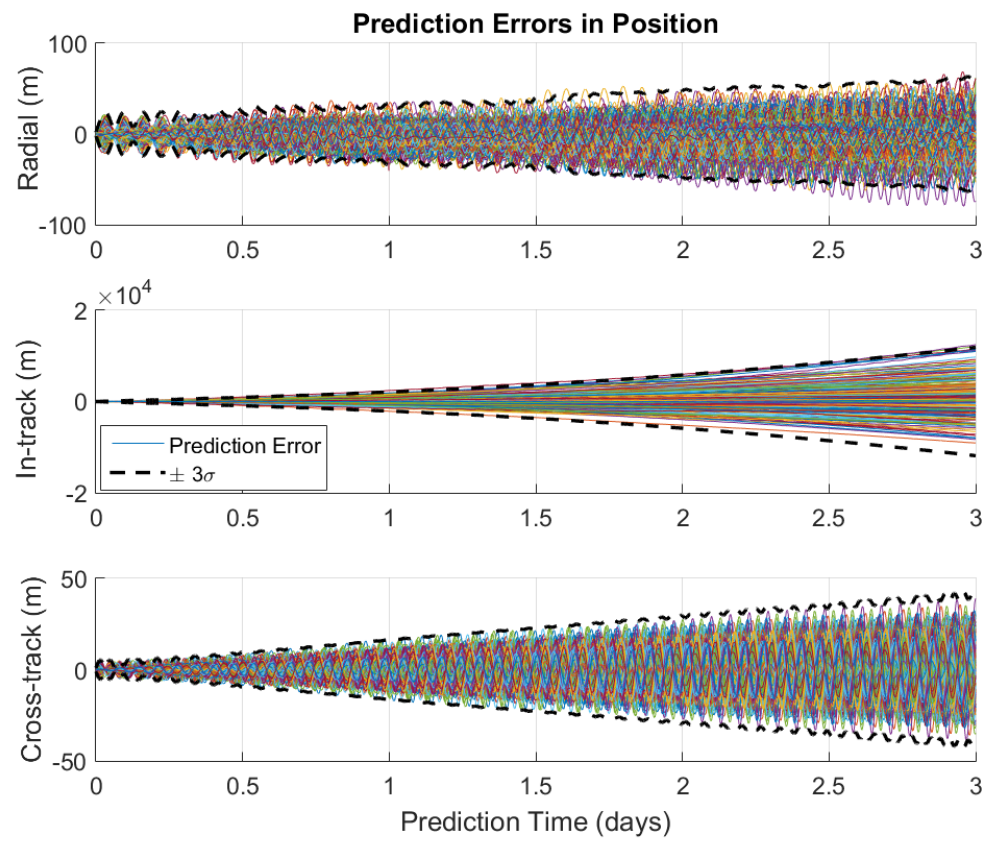

Fig. 4 Predicted ephemeris errors after the removal of outliers.

The overlap ephemeris comparison provide the means to measure the observed level of dispersion (uncertainty) of the predicted ephemeris solutions. Accurate and precise definitive OD solutions result in the reduction of prediction error and their deviations form one solution to the next. In that, the magnitude of the prediction error relates to the accuracy of the definitive OD solution, 
while the amount of the dispersion in a population of the predicted error data relates to the precision (consistency) of the OD process. Figure 5 shows the root sum of squares (RSS) of the overlapped predicted errors with respect to the propagation time in the satellite centered Radial, In-track, and Cross-track (RIC) directions. It also shows the predicted uncertainty in the same coordinates for a handful of selected ephemeris generated by GPM flight operation team (FOT). The curves generated by different FOT files look as if they fall on top of each other at the scale of this plot.

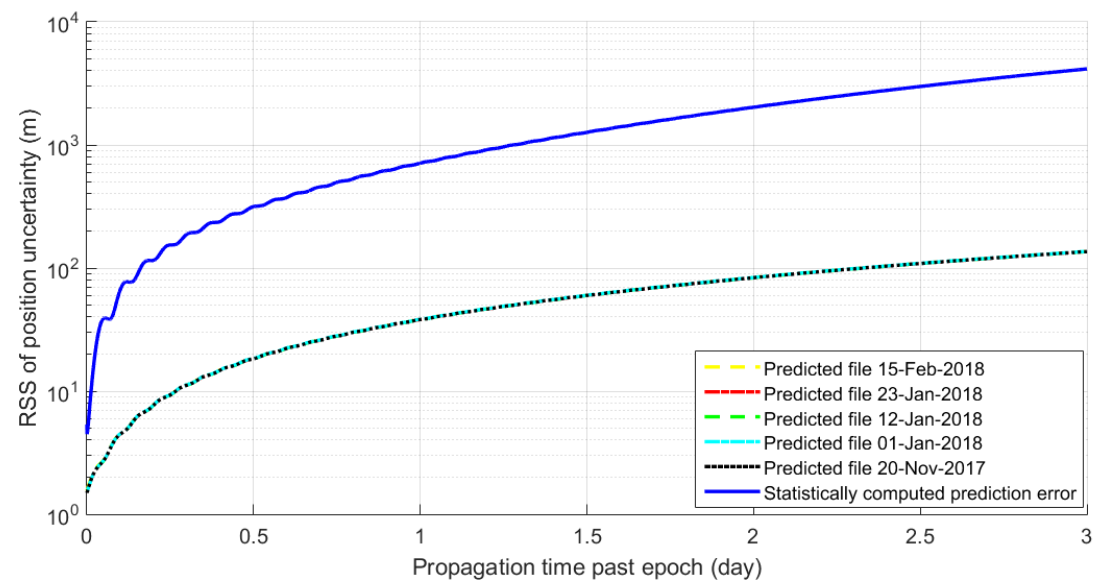

Fig. 5 RSS of the predicted position errors and the FOT predicted position uncertainty.

It is clear that the predicted uncertainty generated by the FOT underestimates the observed level dispersion that exists in the predicted error data, i.e. FOT predicted uncertainty is not a realistic representation of the actual prediction error dispersion. Many factors contribute to this effect; errors in the OD process and dynamical model, drag coefficient, and predicted atmospheric effects are some of such factors that result in an un-realistic ephemeris uncertainty solution. In Section V, we describe the process of scaling the predicted uncertainty via the use of process noise tuning to a realistic level.

\section{B. GOF test of the prediction errors}

A fundamental assumption for the covariance realism process is that the predicted error population follows a Gaussian distribution, which is fully described by the population mean and covariance. In the event that there is significant divergence of the predicted error population from a Gaussian distribution, scaling of the predicted uncertainty (covariance) would not be sufficient to fully describe the behavior of predicted data. A statistical GOF test is implemented to test for the hypothesis that the predicted error data population follows a Gaussian distribution. Let $\mathbf{x}$ be an $n \times 1$ vector drawn from a multivariate Gaussian distribution with mean $\mu_{\mathbf{x}}$ and covariance matrix $P$ that represents the variance-covariance of the distribution, i.e. $\mathbf{x} \sim \mathcal{N}\left(\mu_{\mathbf{x}}, P\right)$. The Mahalanobis distance $d^{2}$ for this distribution is given by [10]

$$
d^{2}=\left(\mathbf{x}-\mu_{\mathbf{x}}\right)^{T} P^{-1}\left(\mathbf{x}-\mu_{\mathbf{x}}\right) .
$$

The quantity $d^{2}$ is a statistical distance between two Gaussian distributions, and follows an $n$ degrees-of-freedom (DoF) $\chi^{2}$ distribution, i.e. $d^{2} \sim \chi_{n}^{2}$.

We adapt the Cramer von Mises (CVM) test statistic $[1,11,12]$ as the metric for the GOF test. This metric measures the sum of squares of the deviations of an empirical cumulative distribution 
function (ECDF) of a sample population $y$ from the cumulative distribution function (cdf) of the hypothesized distribution. The CVM test statistic, denoted by $\omega^{2}$, is as follows

$$
\omega^{2}=m \int_{-\infty}^{\infty}\left[F_{m}(y)-\mathcal{F}(y)\right]^{2} d y,
$$

where $m$ is the sample size, $F_{m}(y)$ is the ECDF derived from the sample population, and $\mathcal{F}(y)$ is the cdf of the hypothesized distribution. CVM method is a member of a more generalized GOF test methods that aim to identify whether or not a given population follows a hypothesized distribution. Further details on this topic is found in Reference 11 among others.

In the case of a discrete sample given by a Monte Carlo type process, the CVM metric may be represented by

$$
\omega^{2}=\frac{1}{12 m}+\sum_{k=1}^{m}\left(\frac{2 k-1}{2 m}-\mathcal{F}\left(y_{k}\right)\right)^{2} .
$$

For the purpose of testing the distribution of the predicted satellite position errors $\delta \mathbf{X}_{3 \times 1}$, the sample population is given by the Mahalanobis distance $\delta \mathbf{X}^{T} \hat{P}^{-1} \delta \mathbf{X}$, where $\hat{P}$ is either derived directly from the population or is given by the propagation of the dynamical model. The $\mathcal{F}(y)$ in this case is the cdf of a $\chi_{3}^{2}$ distribution. The hypothesis that the distribution of the sample follows a $\chi_{3}^{2}$ distribution is rejected, if the $p$-value given by the CVM test statistics falls outside of a $1-\alpha$ confidence interval. We consider the $95 \%$ confidence interval for this analysis, i.e. $\alpha=5 \%$.

The first test is done on the predicted error population alone, in that the covariance matrix is derived directly from the sample population. The purpose of this analysis is to establish whether or not the sample dataset follow a Gaussian distribution. Figure 6 shows the scatter plot of the predicted errors scaled by their corresponding standard deviation in the RIC coordinates after 1, 2, and 3 days of propagation. The plot also shows the corresponding $p$-value of the GOF test result for each scatter plot. Figure 7 shows the cdf of the hypothesized $\chi_{3}^{2}$ distribution and the ECDF derived from the Mahalanobis distance computed from the predicted position errors after 1,2 , and 3 days of propagation. This plot shows a close fit between the hypothesized cdf and the empirical ones. The corresponding $p$-value of the GOF test further suggest a close to perfect fit. Hence, one can safely assume that the predicted error population is un-biased (zero mean) and follows a Gaussian distribution. This also alludes to the fact that any divergence in the Gaussian properties of the predicted error population, when scaled by an externally propagated uncertainty profile, is due to unrealistically sized propagation uncertainty that does not represent the true dispersion of the population.

\section{FOT covariance assessment}

Second set of results involves the GOF test of the predicted error population scaled by the propagated uncertainty provided by the FOT. Figure 8 shows the overlap of the hypothesized $\chi_{3}^{2}$ cdf versus the ECDF provided from the scaled data, at the epoch as well as day 1, 2, and 3 of the propagation. The corresponding GOF test p-values are also noted on the plots. It is easy to see that once the predicted error population is scaled by the FOT provided uncertainty, the resulting distribution diverges from the Gaussian distribution, significantly. Hence, one can conclude with a very high degree of confidence that the FOT provided uncertainty profile is not realistic. Together with the results shown in Figure 5, it is determined that the FOT provided uncertainty underestimates the true prediction uncertainty by a significant amount. We remedy this issue by inflating the propagated covariance via the addition of process noise, detailed in the next section. 

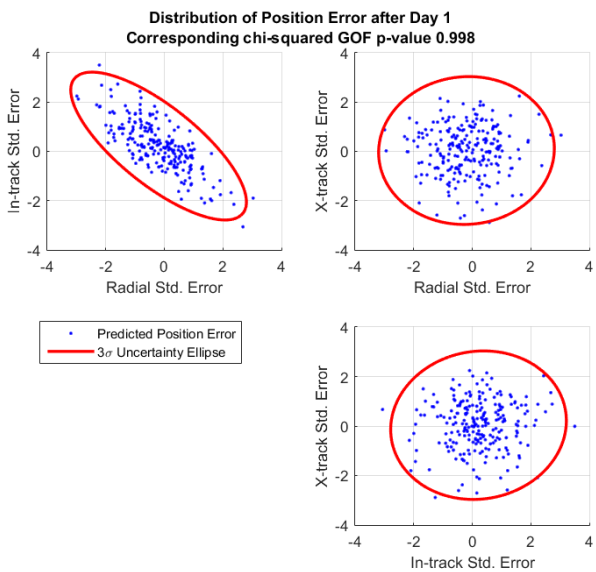

(a) 1 day out
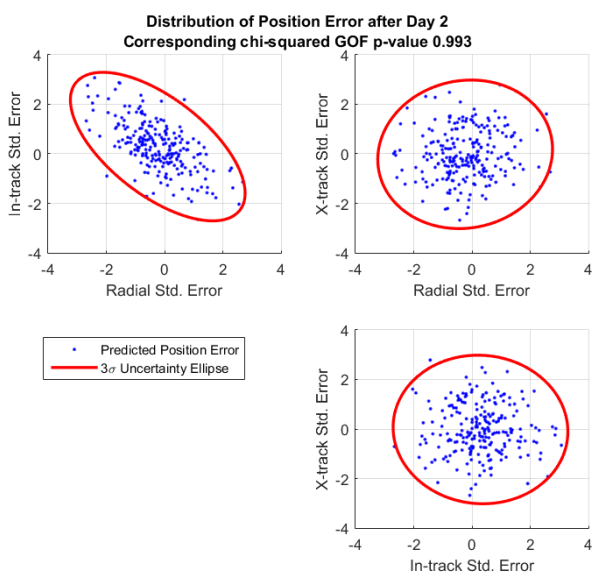

(b) 2 days out
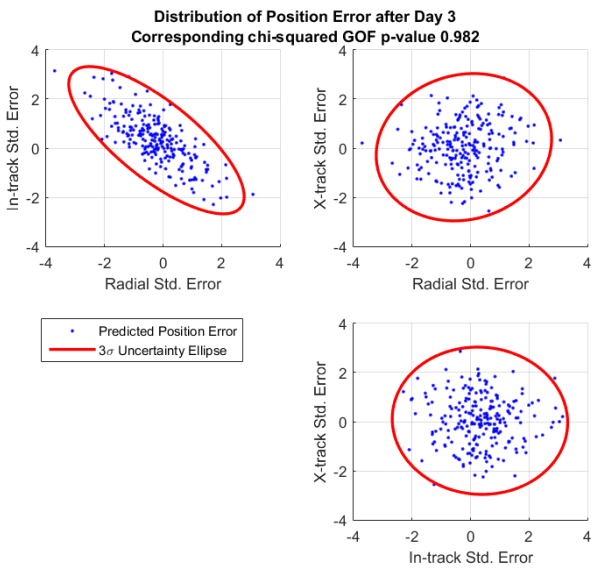

(c) 3 days out

Fig. 6 Scatter plots and $3 \sigma$ dispersion ellipses of the predicted standard errors.

\section{Covariance Scaling via Process Noise}

The previous section showed that the FOT provided propagated uncertainty underestimates the true dispersion of predicted error population, significantly. The approach implemented in this study is to scale the uncertainty to a point that is able to represent the realistic dispersion. The scaling is done by the means of including process noise within the propagated uncertainty. The use of process noise (a.k.a. state noise compensation) $[13,14]$ is commonplace in orbit determination algorithms. The purpose of it is to inflate the uncertainty within the orbit determination arc to account for any unknown and/or unaccounted for forces that act on the object being tracked. It is, however, not as widely used for the purpose of predicted covariance realism. SpaceNav's covariance realism tool utilizes process noise for generating realistic covariance for the predicted GPM trajectories. Others have used this method to generate realistic uncertainty profiles for the Earth Observing Satellite (EOS) constellation [15].

\section{A. Process Noise Tuning and GOF test}

While the application is different, the concept of using process noise in predicted covariance realism is very similar to that of orbit determination process. It is used to inflate the propagated 


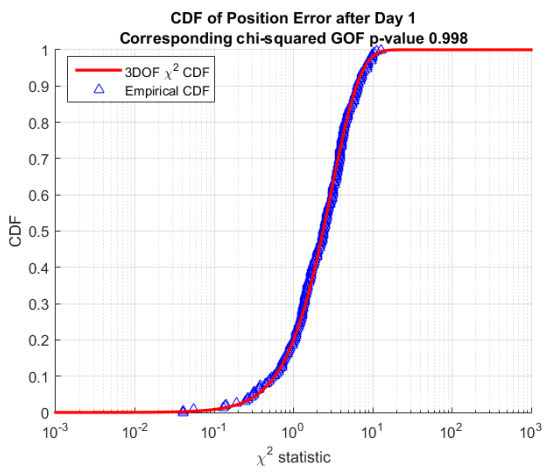

(a) 1 day out

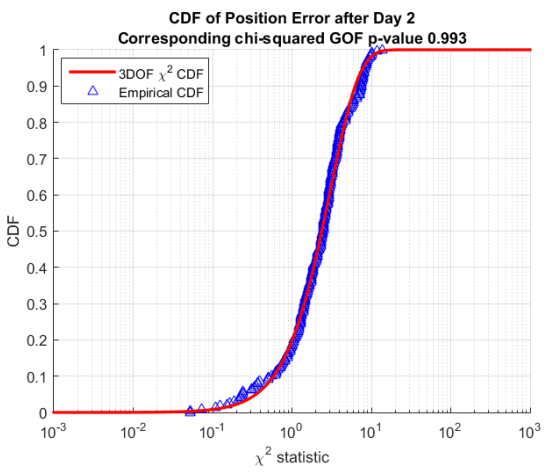

(b) 2 days out

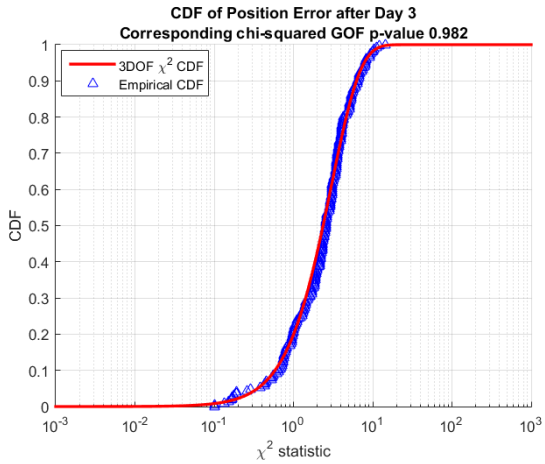

(c) 3 days out

Fig. 7 CDF of the 3-DoF $\chi^{2}$ distribution and the empirical CDF of the overlapped data scaled by population standard deviation.

covariance $\bar{P}$ via the following equation

$$
\bar{P}_{t}=\Phi_{0}^{t} P_{0} \Phi_{t}^{0}+\Gamma_{\Delta t} R Q R^{T} \Gamma_{\Delta t}^{T}
$$

where $P_{0}$ is the state covariance matrix at the epoch of the propagation, $\Phi_{0}^{t}$ is the state transition matrix extending from epoch to time $t$, and $\Gamma$ is the process noise transition matrix for the same time span. The matrix $Q$ contains the process noise acceleration parameters in the satellite centered RIC coordinate system, which are transformed into the propagation frame (often chosen to be an inertial frame) via the coordinate transformation matrix $R$. Process noise acceleration parameters are arranged within the $Q$ matrix as the following.

$$
Q=\left[\begin{array}{ccc}
\sigma_{R}^{2} & 0 & 0 \\
0 & \sigma_{I}^{2} & 0 \\
0 & 0 & \sigma_{C}^{2}
\end{array}\right]
$$

where $\sigma_{R, I}, C$ indicates the amount of the assigned acceleration in the radial, in-track, or cross-track directions, respectively. The accelerations are mapped onto the satellite position and velocity states via the process noise transition matrix given by

$$
\Gamma(\Delta t)=\Delta t\left[\begin{array}{c}
\frac{1}{2} \Delta t I \\
I
\end{array}\right],
$$

where $I$ is a $3 \times 3$ identity matrix.

The next step in the covariance realism process is to adjust the assigned process noise acceleration parameters, such that the resulting scaled propagated uncertainty matches the observed 


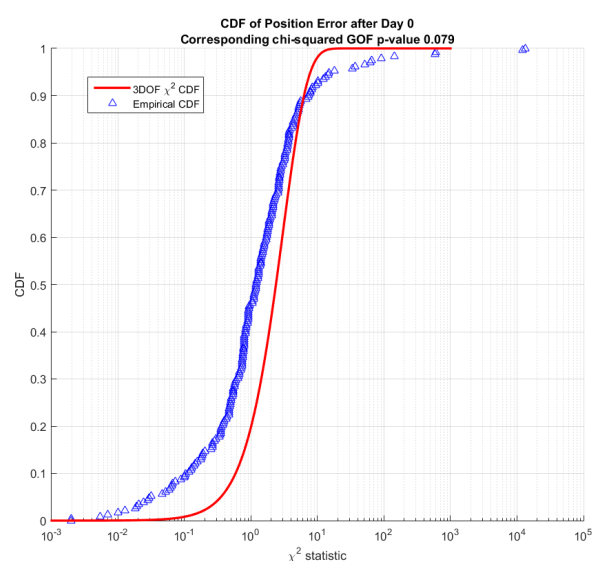

(a) Prediction epoch

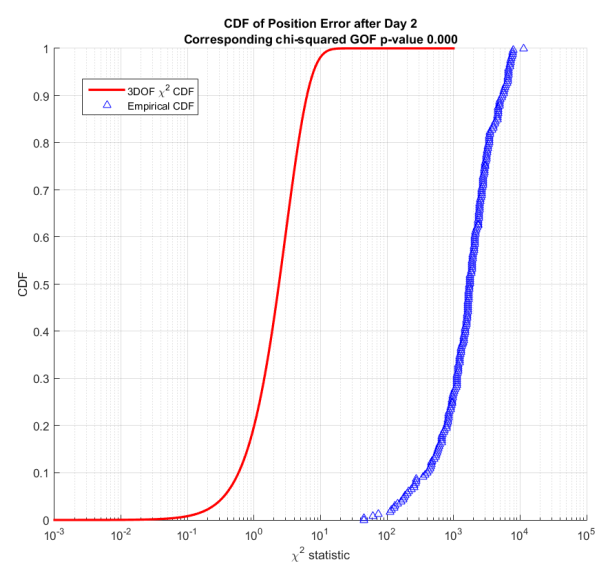

(c) 2 days out

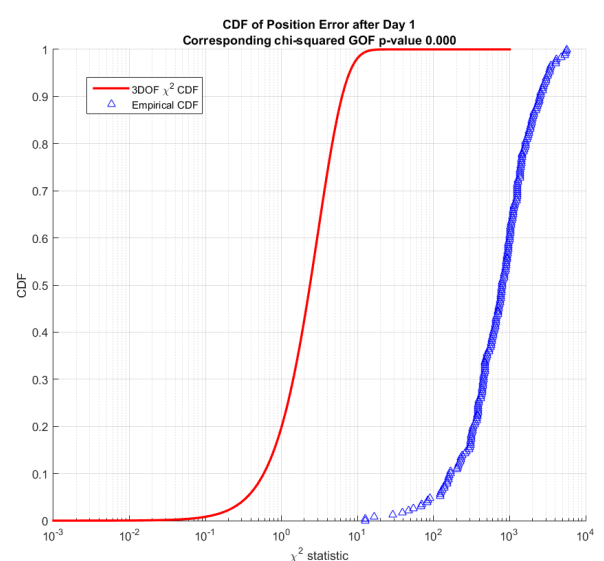

(b) 1 day out

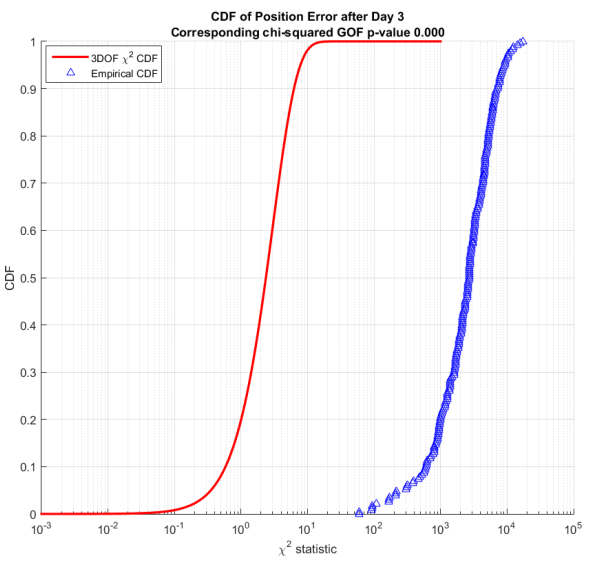

(d) 3 days out

Fig. 8 CDF of the 3-DoF $\chi^{2}$ distribution and the empirical CDF of the overlapped data scaled by the FOT predicted covariance.

predicted trajectory error dispersion. The aim is to tune the process noise parameters such that a certain cost function is minimized. We explore two criteria for the tunning of the parameters, namely mean error and final error. The mean error is given by the RMS of the difference between the scaled propagated uncertainty versus the statistically computed predicted error dispersion averaged over the propagation time. Final error computes the same difference, but averaged over one orbit period at the end of the propagation time. SpaceNav covariance realism tool allows the analyst to tune the process noise parameters against the cost function of choice, manually or automatically using an optimization process. Manual tuning can be a tedious task, which may also become unintuitive at times. This is due to the fact that there are strong cross correlations between the radial and in-track dynamics of the satellite, and changing the process noise acceleration in one direction would affect the uncertainty in the other dimensions as well, which may not be intuitive to the analyst. Furthermore, the manually tuned parameters are subject to the analyst's discretion of the sufficient convergence and may not be consistent from one person to the next. Process noise optimization algorithm removes these issues. In this analysis, we utilize an iterative least squares optimization algorithm to minimize the cost function below a threshold of $1 \%$ error in position uncertainty. Let $\overline{\sigma_{p}}(t)$ be the scaled propagated satellite position uncertainty that is given by the RSS of the square root of the first three diagonal terms of the propagated covariance matrix $\bar{P}$ at time $t$. The observed 
position uncertainty at time $t, \sigma_{o b s}(t)$, is computed by taking the RSS of the statistically generated uncertainty profile in the RIC coordinates using the overlap comparison, described in Section IV. The tuning criterion is given by the percent error $\epsilon_{\sigma}$ in the propagated uncertainty profile computed over a particular propagation window (mean vs. final error), i.e.

$$
\epsilon_{\sigma}=\frac{1}{N} \sum_{t=t_{0}}^{t_{e n d}} \frac{\sigma_{o b s}(t)-\overline{\sigma_{p}}(t)}{\sigma_{o b s}(t)} \times 100,
$$

where $N$ is the total number of the time steps within the propagation period that is considered for the error criterion. The tuning algorithm aims to find a set of process noise parameters that minimizes the cost function

$$
J=\frac{1}{2} \epsilon_{\sigma}^{2}
$$

The least squares solution of the algorithm is given by

$$
\hat{\sigma}=\left(H^{T} H\right)^{-1} H \epsilon_{\sigma}
$$

where

$$
H=\frac{\partial \epsilon_{\sigma}}{\partial \sigma},
$$

and $\sigma=\left[\begin{array}{lll}\sigma_{R} & \sigma_{I} & \sigma_{C}\end{array}\right]^{T}$. The sensitivity matrix $H$ is computed numerically via a finite differencing method.

The least squares method solved for the linearized version of what could be a fairly complex cost function. Hence, we iterate the solution until a convergence tolerance is met, which is set to be $1 \%$. Figure 9 shows the value of the cost function with the final error criterion for a range of process noise parameters shown in $\log _{10}$ scale. The vertical axes represent the process noise in the in-track direction, and the horizontal axes represent the radial component. Each contour plot corresponds to the indicated value of cross-track process noise parameter. The contour plots show that the cost function is smooth within the domain that is considered with clear absolute minimum region. This is a favorable characteristic that allows for the least squares method to zoom onto the optimum solution after few iterations.

The optimization method was used to tune the process noise and generate scaled propagated covariance for all of the ephemeris files considered in Section IVB. Following that, a GOF test was performed to assess whether or not the scaled propagated uncertainty represent the realistic predicted error dispersion. Figure 10 shows the p-value of the GOF test of the overlapped data scaled by the propagated covariance over the propagation time. Figure 10(a) shows this for the case when the process noise tuning was performed based on the final error tuning criterion. Figure 10(b) is the case for the mean error tuning metric. First, it should be noted that a majority of the propagation period passes the GOF test with a $95 \%$ confidence level for both tuning criteria. Hence the scaled propagated covariance via the injection of the process noise is able to represent the realistic covariance of the overlapped data. More interestingly, these plots show that the maximum confidence level is achieved at the end of the propagation period for the final error criterion, and midway through the propagation for the mean error criterion. This result conforms with the intuition, in that the tuning algorithm effectively aims to minimize the cost function at a certain time within the propagation window subject to the error criterion being considered. Figure 11 shows the overlap of the hypothesized $\chi_{3}^{2} \mathrm{cdf}$ and the ECDF generated from the overlapped data population scaled by the propagated uncertainty, when tuned to the final error metric. This plot shows a close agreement 

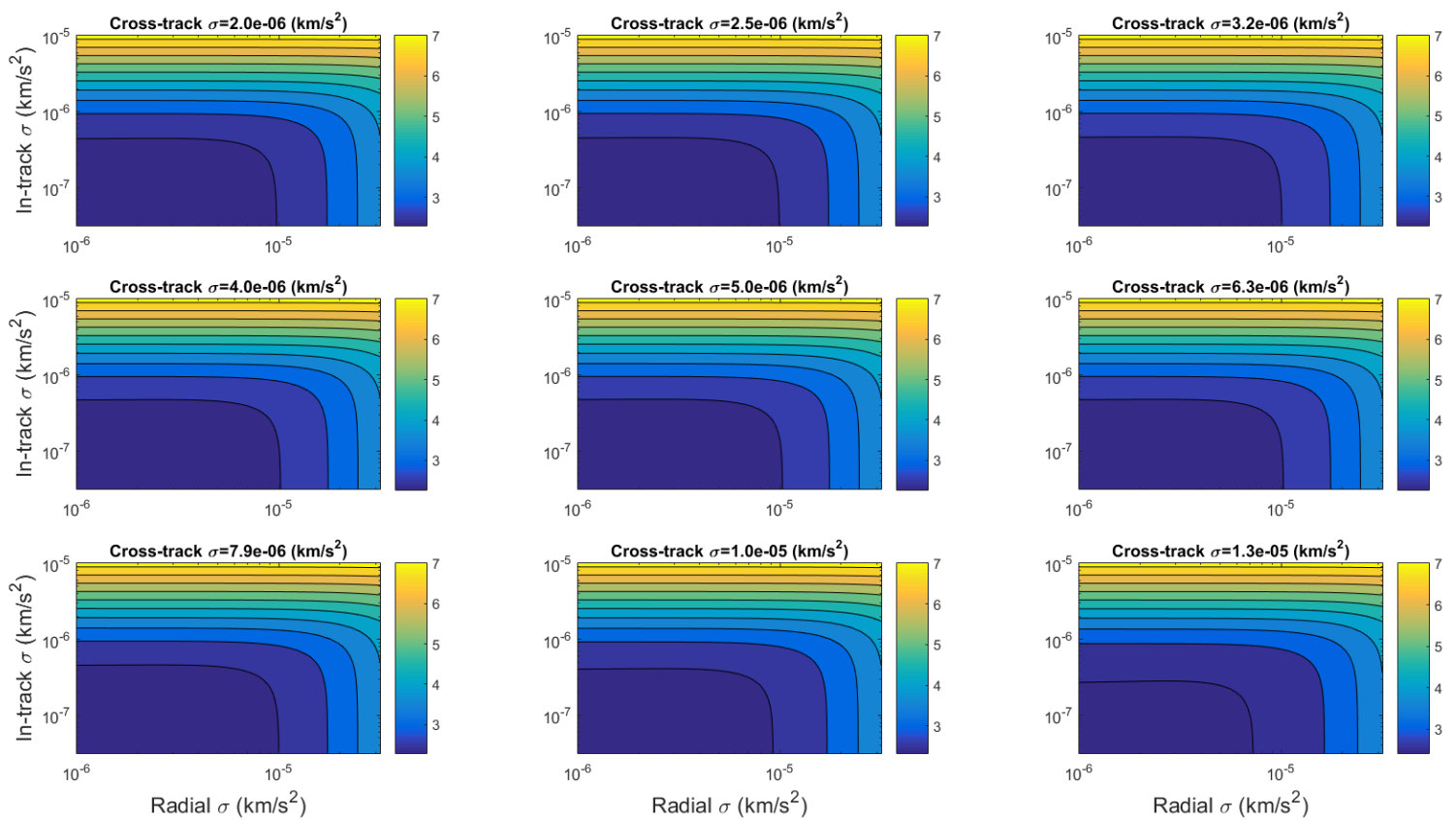

Fig. 9 Process noise tuning cost function contour plots. Final error criterion.

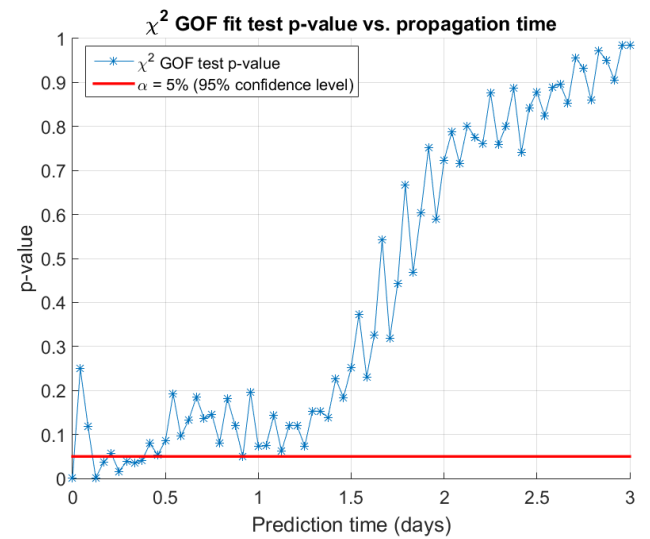

(a) Final Difference

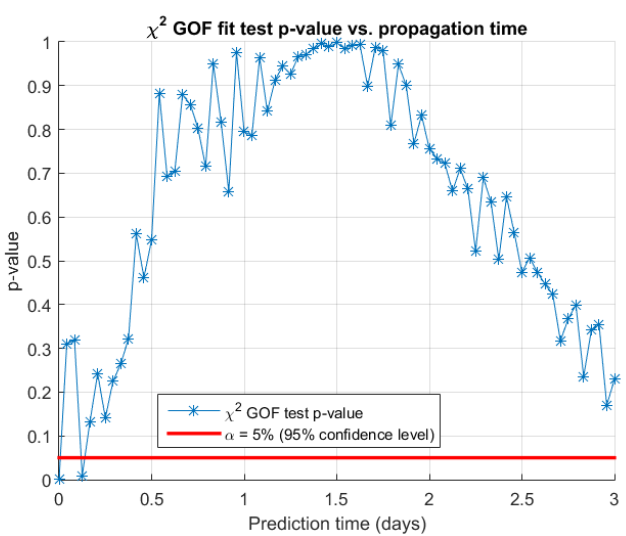

(b) Mean Difference

Fig. 10 p-value of the 3-DoF $\chi^{2}$ GOF test for overlapped data scaled by corrected predicted covariance; $10(\mathrm{a})$ is tunned to minimize the final difference, while $10(\mathrm{~b})$ minimizes the mean difference.

between the ECDF derived from the data and the hypothesized $\chi_{3}^{2}$ cdf after 1,2 , and 3 days of propagation.

According to these results, it is suggested to tuned the process noise parameters to a final error criterion at the prediction time of interest, whether it being a 3 days out or shorter. That is when the best performance in the covariance realism is achieved. To this end, it is of interest to perform a comparative analysis of the process noise tuning to different time scales, and quantify the sensitivity of the process noise parameters to different tuning spans if the mission operations decides to do so. 


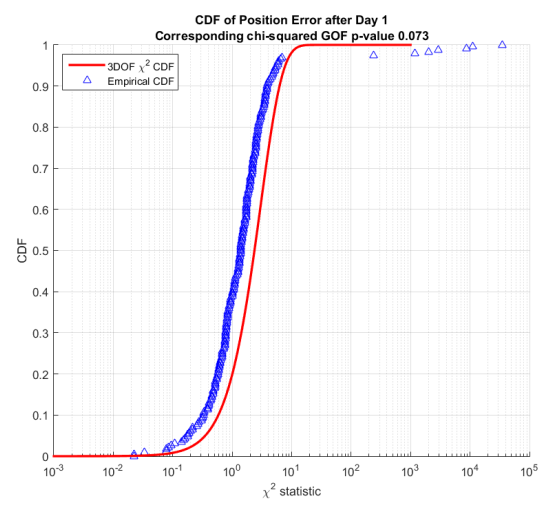

(a) 1 day out

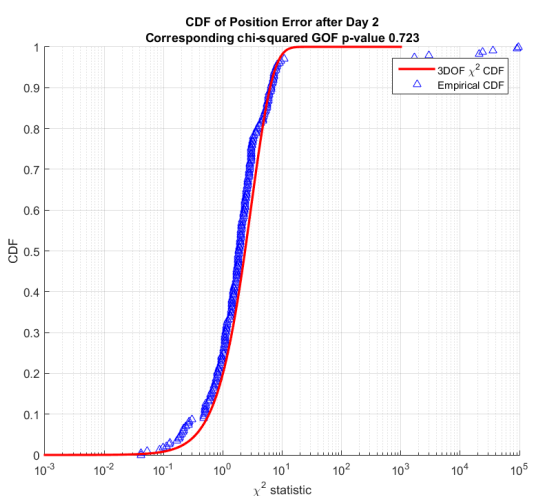

(b) 2 days out

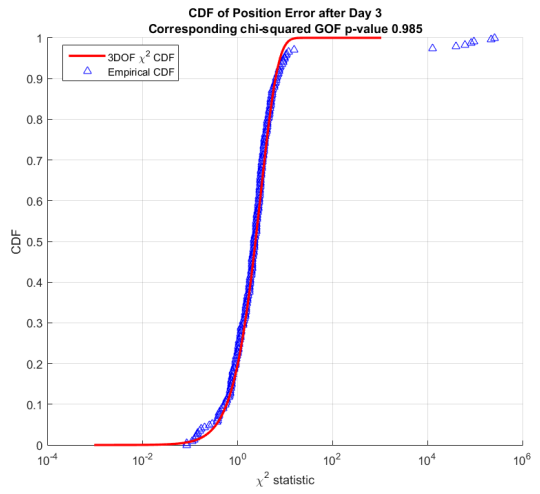

(c) 3 days out

Fig. 11 CDF of the 3-DoF $\chi^{2}$ distribution and the empirical CDF of the overlapped data scaled by the corrected predicted covariance.

\section{B. Process noise tuning sensitivity analysis}

Previously, we looked at tuning of the process noise for a 3-day prediction span, whether using a mean error or a final error criterion. Recall that there is a specific point along the propagation time (see Figure 10), where the predicted error population scaled to the propagated covariance via process noise exhibits an optimum Gaussian property. This point corresponds to the tuning span and the type of the tuning metric that is considered. Hence, the flight operations team might have an interest in tuning the process noise to different tuning spans, depending on how many days in the future the time of closest approach (TCA) of an event of interest is. This section looks at the sensitivity of the tuning parameters to different tuning spans. We perform the tuning for 1,2 , and 3 days out and consider the final error metric for this analysis.

Figure 12 shows the results of the analysis. Figure 12(a) shows the process noise parameter values when tuned to different time spans. It is very intuitive to see that the process noise parameters increase in the in-track and cross-track directions as the tuning span increases. However, the plot also shows that the radial component decreases by the increased tuning span. This may seem un-intuitive at first. However, it can be explained by the strong correlation the exists between the radial and in-track components and that the increase in the in-track component more than compensates for the decrease in the radial direction. Finally, Figure 12(b) shows the propagated uncertainty profiles for three tuning spans superimposed on the uncertainty profile that is computed statistically from the overlap comparison analysis. The " $\mathrm{x}$ " markers shows the points where the two uncertainty profiles are matched. 


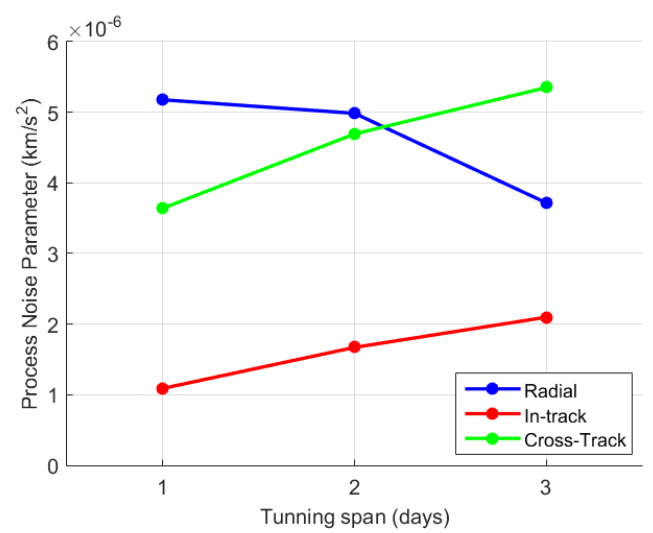

(a)

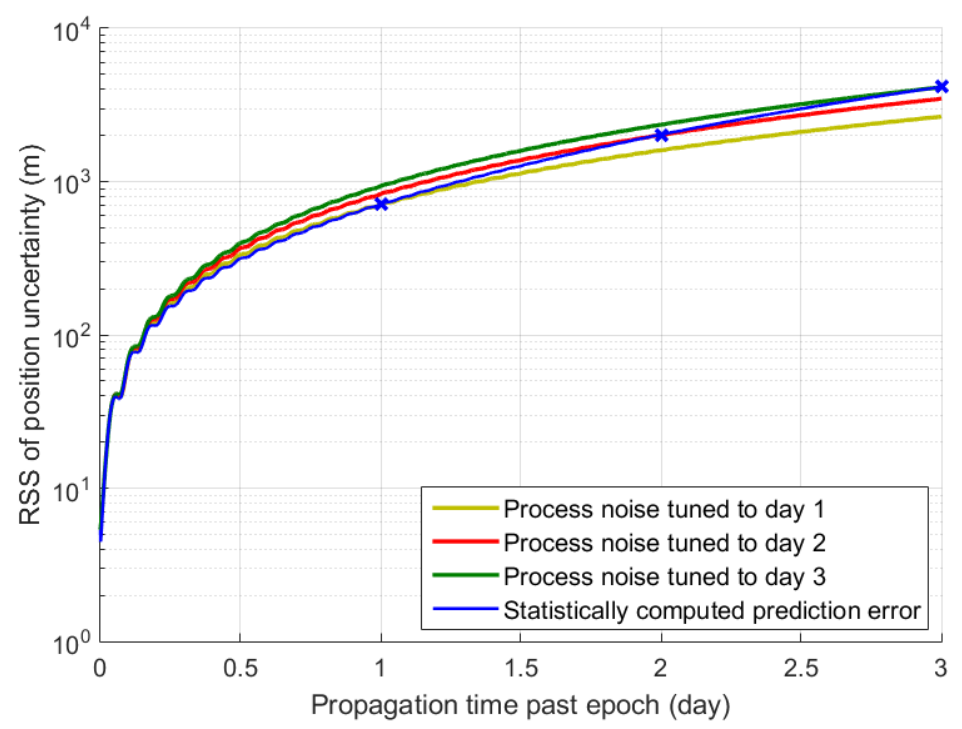

(b)

Fig. 12 Process noise tuning parameters vs. the tuning span.

\section{Maneuver Uncertainty Implementation}

GPM spacecraft performs frequent trim maneuvers as part of the regular orbit maintenance procedure. Collision avoidance maneuvers are also planned and executed as part of the collision avoidance strategy. Such maneuvers are planned multiple days in advanced and are delivered to SpaceNav in the form of predicted maneuver plan files. Furthermore, the planned maneuvers are included within the nominal predictive ephemeris files generated by the GPM flight operations team. As a result, the covariance realism tool must take into account the expected level of error in the planned maneuver when generating the predicted covariance, if one happens to be within the propagation time span. To do this, we performed a comprehensive analysis of the past maneuver execution performance by the GPM spacecraft. The result of such analysis provides us with a measure of the expected maneuver error level in the future.

The GPM spacecraft performs along or anti-velocity direction maneuvers using a set of 12 thrusters located on the forward and aft side of the spacecraft bus. The spacecraft may be in a $0^{\circ}$ or $180^{\circ}$ yaw orientation due to the solar beta angle configuration at the time of the maneuver execution. The combination of the forward vs. aft thruster sets as well as the yaw orientation 
of the spacecraft results in four different maneuver configurations, which ultimately result in two overall outcomes; posigrade vs. retrograde velocity changes. Table 1 summarizes the four different combinations of the thruster set and the resulting maneuver outcomes numbered 1 through 4 . For the purpose of maneuver performance analysis, we categorized into two main types based on the thruster set that is used; type-I uses the aft thrusters and type-II uses the forward thruster set. An analysis of the past maneuver performance for the GPM spacecraft was performed. The maneuver performance data was provided to SpaceNav by the FOT. The data included all of the maneuvers that are performed by the GPM spacecraft until very recently. It included a total of 53 maneuvers, 25 type-I and 28 type-II.

Figure 13 shows the maneuver percent error versus delivered $\Delta V$ magnitude. The maneuver error percentage is given by

$$
\epsilon_{\text {mnvr }}=\frac{\left|\Delta \mathbf{V}_{\text {achieved }}-\Delta \mathbf{V}_{\text {planned }}\right|}{\left|\Delta \mathbf{V}_{\text {achieved }}\right|} \times 100
$$

This figure shows that there is no significant correlation between the performed maneuver errors and their corresponding magnitudes. It also shows that there exist three outliers in the data, one in the type-I and 2 in the type-II maneuvers. The type-I outlier corresponds to a small test maneuver right after the launch and deployment of the spacecraft. Type-II outliers correspond to drag makeup maneuvers (DMU) number 3 and 4, respectively. These are also considered as outliers since they fall outside of the $\pm 3 \sigma$ bounds, and both are executed early in the mission. Other analyses were also performed to look at the correlations between the maneuver error magnitude as a function of time, and correlation of thruster duty cycle versus the maneuver magnitude. According to these analysis, it was determined that there is a $2.43 \%$ error in the execution of the type-I maneuvers, and a $1.05 \%$ in the type-II ones. These values are given by the RMS of the maneuver execution error percentage after the removal of the outliers. While the error values are derived from the available maneuver performance data, it is worth to note that there are not many data points available to draw a more statistically significant conclusion. Similar analysis should be conducted each time there is a new maneuver execution data to update the estimated error values. Furthermore, in the previous set of analyses, it is assumed that the maneuver pointing is known exactly, i.e. there are no thrust vector pointing errors. This assumption was communicated between SpaceNav and FOT and it was concluded to be a reasonable assumption.

Table 1 GPM On-orbit Maneuver Type Combinations

\begin{tabular}{clll}
\hline \hline Maneuver Type S/C Yaw Orientation & Active thruster Set & Maneuver Outcome \\
\hline 1 & forward facing & aft ( thrusters 1-8 ) & Posigrade \\
\hline 2 & backward facing & fwd (thrusters 9-12 $)$ & Posigrade \\
\hline 3 & forward facing & aft (thrusters 1-8 ) & Retrograde \\
\hline 4 & backward facing & fwd (thrusters 9-12) & Retrograde \\
\hline \hline
\end{tabular}

The RMS of the past maneuver performance errors is taken to be a first order measure of the uncertainty in the maneuver execution. The maneuver execution uncertainty is injected into the SpaceNav generated realistic propagated covariance, if the predicted maneuver epoch happens to fall within the propagation time period. SpaceNav covariance realism tool implements the Gates model [16] to map the uncertainty in the maneuver onto the spacecraft state parameters. The Gates model takes into account both the maneuver magnitude and pointing errors, and is used operationally on different missions including Cassini $[17,18]$ spacecraft maneuver planning and execution. According to the Gates model, a maneuver execution error expressed in a coordinate 


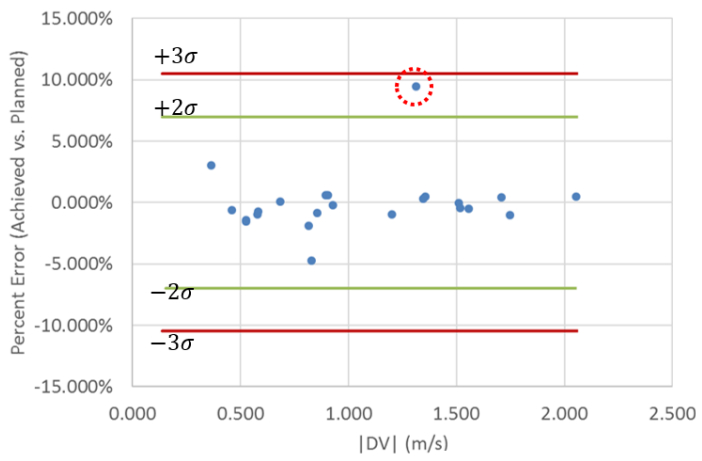

(a) Type I

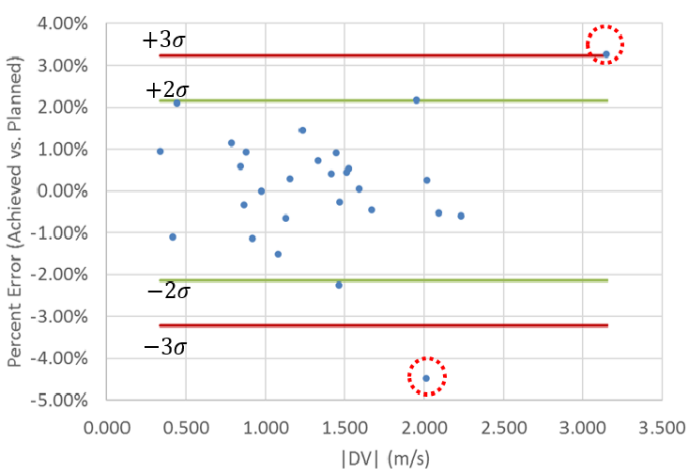

(b) Type II

Fig. 13 Maneuver percent error versus delivered $\Delta V$ magnitude. Figure 13(a) corresponds to the performance results for the thruster set 1-8. Figure 13(b) shows the results for the thruster set 9-12.

frame whose 1st axis is aligned along the $\Delta \mathbf{V}$ vector is given by

$$
\delta \Delta \mathbf{V}=\sqrt{\varepsilon_{1}^{2}+\varepsilon_{2}^{2}|\Delta \mathbf{V}|^{2}} \hat{\mathbf{e}}_{1}+\sqrt{\varepsilon_{3}^{2}+\varepsilon_{4}^{2}|\Delta \mathbf{V}|^{2}} \hat{\mathbf{e}}_{2}+\sqrt{\varepsilon_{3}^{2}+\varepsilon_{4}^{2}|\Delta \mathbf{V}|^{2}} \hat{\mathbf{e}}_{3},
$$

where $\varepsilon_{1}$ and $\varepsilon_{3}$ correspond to biases in the maneuver magnitude and pointing, respectively. Variables $\varepsilon_{2}$ and $\varepsilon_{4}$ correspond to proportional errors in the maneuver magnitude and pointing. The model coordinates are defined by the $\widehat{\mathbf{e}_{1} \mathbf{e}_{2} \mathbf{e}_{3}}$ orthogonal triad, such that

$$
\hat{\mathbf{e}}_{1}=\frac{\Delta \mathbf{V}}{|\Delta \mathbf{V}|}, \quad \hat{\mathbf{e}}_{2}=\frac{\left[\begin{array}{lll}
{[} & 0 & 1
\end{array}\right]^{T} \times \hat{\mathbf{e}}_{1}}{\left|\left[\begin{array}{lll}
0 & 0 & 1
\end{array}\right]^{T} \times \hat{\mathbf{e}}_{1}\right|}, \text { and } \hat{\mathbf{e}}_{3}=\hat{\mathbf{e}}_{1} \times \hat{\mathbf{e}}_{2} .
$$

The resulting maneuver covariance matrix is then given by

$$
P_{m n v r}^{\mathbf{e}}=\left[\begin{array}{ccc}
\varepsilon_{1}^{2}+\varepsilon_{2}^{2}|\Delta \mathbf{V}|^{2} & 0 & 0 \\
0 & \varepsilon_{3}^{2}+\varepsilon_{4}^{2}|\Delta \mathbf{V}|^{2} & 0 \\
0 & 0 & \varepsilon_{3}^{2}+\varepsilon_{4}^{2}|\Delta \mathbf{V}|^{2}
\end{array}\right],
$$

where superscript e signifies the maneuver coordinate frame, and is transformed into the propagation coordinate frame via

$$
P_{m n v r}=[R] P_{m n v r}^{\mathbf{e}}[R]^{T}
$$

where $[R]$ defines the mapping from the maneuver frame into the propagation frame. The maneuvers are treated as impulsive velocity changes that occur at the mid point of the scheduled burn time. The resulting maneuver uncertainty is incorporated as an inflation in the propagated state covariance via the following equation

$$
P^{+}=P^{-}+\left[\begin{array}{cc}
0 & 0 \\
0 & P_{m n v r}
\end{array}\right]
$$

where $P^{-}$and $P^{+}$are the propagated state covariance matrices pre and post-maneuver. In the GPM spacecraft maneuver uncertainty implementation, we assume that there are no pointing errors, and that the magnitude error is solely proportional to the $\Delta \mathbf{V}$ (there is no significant bias according the Figure 13), i.e. $\varepsilon_{1}, \varepsilon_{3}$, and $\varepsilon_{4}$ are set equal to zero.

Figure 14 shows the result of the implementation of the maneuver uncertainty in the propagated uncertainty generated by SpaceNav and compares it to the predicted uncertainty provided by the 
GPM FOT. It corresponds to a posigrade drag makeup maneuver with a planned magnitude of 0.459 $\mathrm{m} / \mathrm{s}$ at 09/15/17 14:31:28 UTC. The FOT predicted ephemeris starts at 09/14/2017 00:00:00 UTC. The SpaceNav propagated ephemeris starts from the same epoch and includes the same predicted maneuver. Table 2 summarizes the force model parameters that were used for the propagation. First, note that there is an unrealistic dip in the in-track uncertainty right before the maneuver

Table 2 Numerical Propagation Force Model and Related Parameters.

\begin{tabular}{ll}
\hline \hline Propagation Epoch & $09 / 14 / 2017$ 00:00:00.000 UTC \\
Numerical Propagator & Dormand-Prince 8(7) variable step size \\
Force Model & \\
Central Body & Earth \\
Geopotential & EGM96 $30 \times 30$ model \\
Atmospheric Model & Jacchia-Roberts \\
Drag and SRP force & Cannonball model \\
Third Body & Sun and Moon (point mass) \\
Process Noise & None \\
Predicted Maneuver & \\
Epoch & $09 / 15 / 1714: 31: 28.000$ UTC \\
Type & Posigrade \\
Magnitude & $0.459 \mathrm{~m} / \mathrm{s}$ \\
Magnitude Error & $2.43 \% \times|\Delta \mathrm{V}|$ \\
Pointing Error & None \\
\hline \hline
\end{tabular}

execution time in the predicted uncertainty provided by FOT. This was identified as an artifact of the orbit determination software used for generating the propagated covariance, according to the communications between SpaceNav and GPM FOT. Second, the plot shows that the SpaceNav generated covariance is larger than the FOT generated one especially in the in-track direction. This may partly be due to the initial dip that occurs in the FOT prediction covariance. However, the exact comparison of the two methods is not feasible since SpaceNav does not have access to details of all the processes that are used to generate this covariance profile.

A verification analysis is performed to test the SpaceNav maneuver uncertainty implementation method. In that, we perform a Monte Carlo simulation, where a total of 6,000 propagated trajectories are generated starting from the epoch of the FOT predicted ephemeris shown in Figure 14. The initial state of each propagation is perturbed from the FOT provided epoch state by a vector that follows a Gaussian distribution of zero mean and covariance matrix equal to FOT provided epoch covariance. Each trajectory is propagated to the maneuver time. At that time an impulsive velocity change that is realized from a Gaussian distribution with a mean equal to the maneuver magnitude and a standard deviation equal to the proportional error in the maneuver magnitude (see Table 1) is applied to the satellite velocity. Then, the post maneuver state is propagated forward until the end of the propagation time. Tabel 3 summarizes the Monte Carlo simulation parameters. Each propagation uses the same force model as the one specified in Tabel 2. Figure 15 shows the RSS of the propagated position errors derived from the population of Monte Carlo propagations at each time from the epoch until day 14 of the propagation. It also shows the RSS of the position uncertainty generated by SpaceNav CRT and the one provided by the FOT predictive ephemeris file, i.e. the RSS of the RIC components shown on Figure 14. This plot shows that the propagated position uncertainty generated by the SpaceNav CRT matches the Monte Carlo simulation results very closely. The plot also shows that the FOT generated uncertainty, significantly under-estimates 

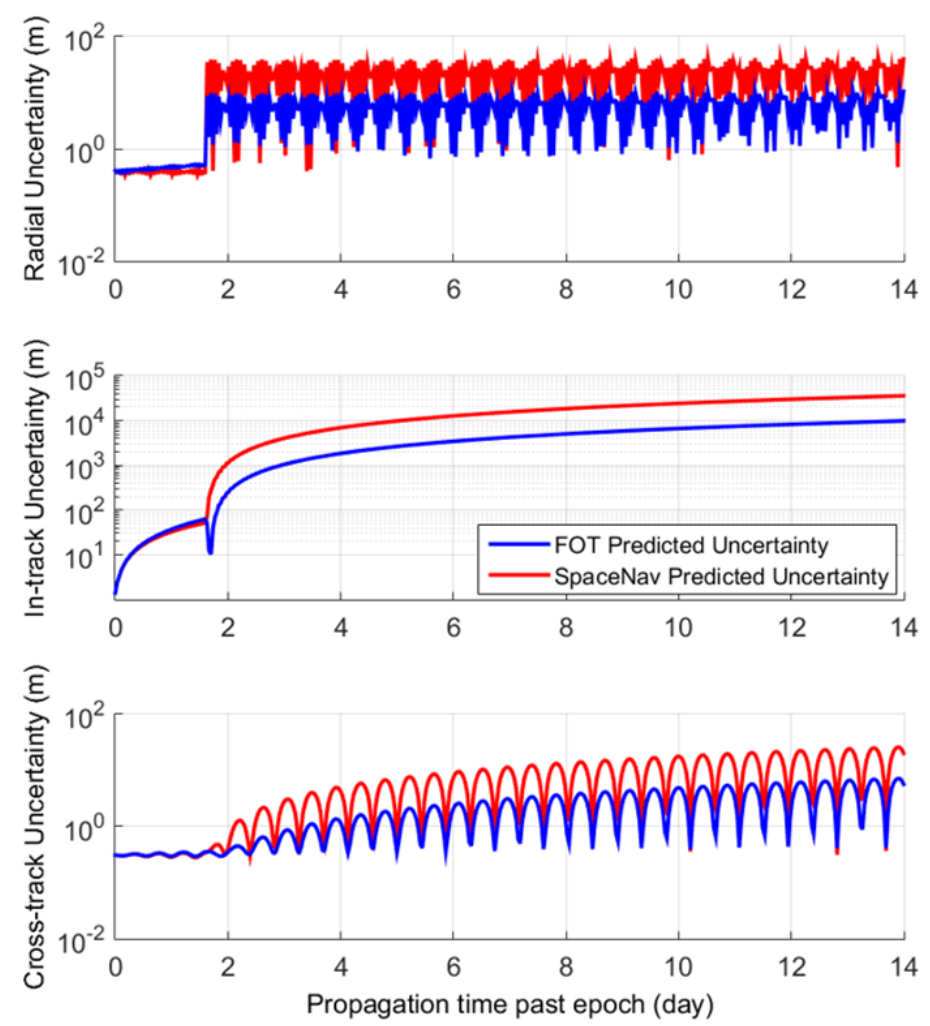

Fig. 14 Propagated uncertainty including a maneuver. Predicted ephemeris epoch is 09/14/2017 00:00:00 UTC. Maneuver occurs at 09/15/17 14:31:28 UTC.

Table 3 Maneuver Monte Carlo Simulation Settings.

\begin{tabular}{ll}
\hline \hline Sample Size & 6,000 \\
Initial State Error & $\sim \mathcal{N}\left(0, P_{0}\right)$, \\
& where $P_{0}=$ FOT predicted file epoch covariance. \\
Predicted Maneuver & \\
Epoch & $09 / 15 / 1714: 31: 28.000 \mathrm{UTC}$ \\
Type & Posigrade \\
Magnitude & $\sim \mathcal{N}\left(0.459, \sigma_{\Delta V}^{2}\right) \mathrm{m} / \mathrm{s}$, \\
& where $\sigma_{\Delta V}=2.43 \% \times|\Delta \mathbf{V}|$. \\
\hline \hline
\end{tabular}

the propagated uncertainty both pre and post-maneuver. Note that there is a difference between the position uncertainty provided by the Monte Carlo simulation versus that provided by the SpaceNav CRT. This difference is due to the fact that the CRT covariance propagation is done using a linear mapping of the covariance matrix with zero process noise, while the Monte Carlo results are derived from direct non-linear propagations. The seemingly large difference is further pronounced by the use of logarithmic scale in the vertical axis of the plot. Implementation of a proper value of process noise, as specified in the previous section, will remedy the difference.

\section{Conclusion}

A comprehensive analysis of the predicted uncertainty profile of NASA's Global Precipitation Measurement (GPM) spacecraft is discussed. GPM spacecraft is in a lower earth orbit just below 


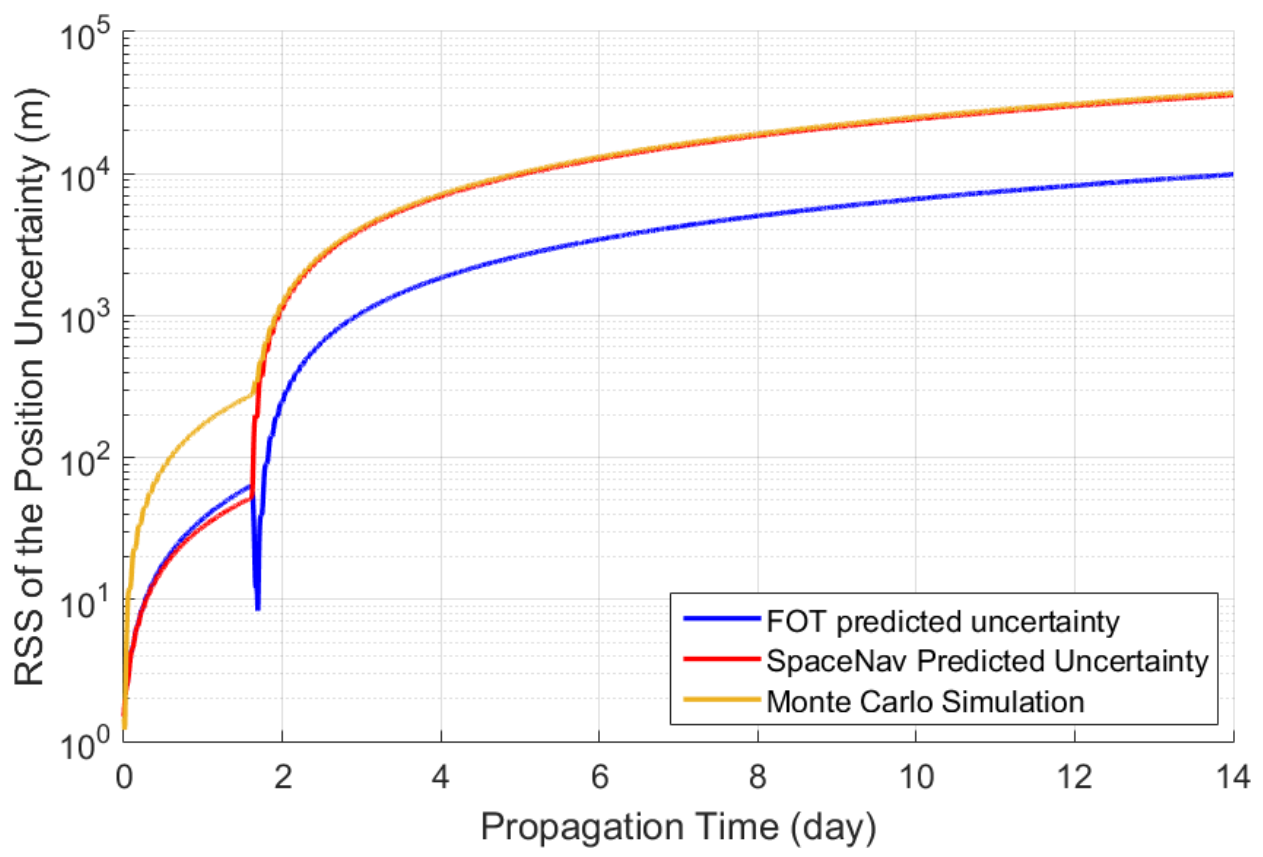

Fig. 15 RSS of the propagated position uncertainty provided by the Monte Carlo simulation, SpaceNav CRT, and FOT predicted ephemeris file, with the inclusion of the maneuver uncertainty.

the altitude of the International Space Station (ISS) orbit. Accurate knowledge of the predictive trajectory uncertainty is crucial for effective close approach analysis of the space debris as well as occasional small-sat deployments from the ISS with less predictable trajectories that may cross the GPM orbit environment. Analysis of the pass two plus years of GPM definitive and predictive ephemeris files revealed that the propagated uncertainty generated by the GPM flight operations software tends to underestimate the true level of predictive trajectory dispersion, by a significant amount. The process of covariance realism carried out by SpaceNav was outlined through the various steps of the data collection, overlap comparative analysis, process noise tuning, and realistic covariance file generation. A goodness-of-fit test was carried out to test for the Gaussian distribution hypothesis of the predictive trajectory error population, when those errors are scaled by the GPM provided uncertainty, and when they are scaled by the realistic covariance generated by the SpaceNav covariance realism tool. It was shown that the predictive trajectory errors do follow a Gaussian distribution, while the predicted covariance profile provided by the GPM operational software moves the data away from a Gaussian distribution. It was further shown that the Gaussian distribution assumption was again valid after the propagated uncertainty profile was corrected via the SpaceNav covariance realism method.

The paper further discussed an analysis of the GPM spacecraft past maneuver performance, and the method that is used to incorporate maneuver uncertainty into the propagated realistic covariance. This method was validated via a Monte Carlo simulations. Results from this study show that scaling the predicted covariance via process noise is a simple and low cost method to produce uncertainty profiles that represent the realistic level of dispersion in the predicted trajectories for SSA applications. Future work will look at the implementation of non-linear propagation methods such as sigma-point propagations and Gaussian mixture models to produce uncertainty profiles that are valid for longer propagation time spans. 


\section{Acknowledgement}

The corresponding author would like to thank the GPM flight operation team members for providing the author with required data.

\section{References}

[1] Alfriend, K. and Park, I., "When Does the Uncertainty Become Non-Gaussian," Advanced Maui Optical and Space Surveillance Technologies Conference, Sept. 2016, p. 46.

[2] Alspach, D. and Sorenson, H., "Nonlinear Bayesian estimation using Gaussian sum approximations," IEEE Transactions on Automatic Control, Vol. 17, No. 4, Aug 1972, pp. 439-448.

[3] Jah, M. and Kelecy, T., "Orbit Determination Performance Improvements for High Area-to-Mass Ratio Space Object Tracking Using an Adaptive Gaussian Mixtures Estimation Algorithm," 60th International Astronautical Congress, 2009.

[4] Horwood, J. T. and Poore, A. B., "Adaptive Gaussian Sum Filters for Space Surveillance," IEEE Transactions on Automatic Control, Vol. 56, No. 8, Aug 2011, pp. 1777-1790.

[5] Horwood, J. T. and Poore, A. B., "Gauss von Mises Distribution for Improved Uncertainty Realism in Space Situational Awareness," SIAM/ASA Journal on Uncertainty Quantification, Vol. 2, No. 1, 2014, pp. 276-304.

[6] Jones, B. A., Doostan, A., and Born, G. H., "Nonlinear Propagation of Orbit Uncertainty Using NonIntrusive Polynomial Chaos," Journal of Guidance, Control, and Dynamics, Vol. 36, No. 2, 2013, pp. 430-444.

[7] Foster, Jr., J. L. and Frisbee, J. H., "Comparison of the Exclusion Volume and Probability Threshold Methods for Debris Avoidance for the STS Orbiter and International Space Station," Tech. Rep. NASA/TP?2007?214751, NASA Johnson Space Flight Center, Houston, TX, May 2001.

[8] Foster, Jr., J. L., "The analytic basis for debris avoidance operations for the International Space Station," Space Debris, edited by H. Sawaya-Lacoste, Vol. 473 of ESA Special Publication, Oct. 2001, pp. 441-445.

[9] Chan, F. K., Spacecraft Collision Probability, The Aerospace Corporation, El Segundo, CA, 2008.

[10] Mahalanobis, P. C., "On the generalised distance in statistics," Proceedings of National Institute of Science, India, Vol. 2, April 1936, pp. 49-55.

[11] Horwood, J. T., Aristoff, J. M., Singh, N., Poore, A. B., and Hejduk, M. D., "Beyond covariance realism: a new metric for uncertainty realism," Proceedings of the SPIE, Signal and Data Processing of Small Targets, Baltimore, MD, Vol. 9092, 2014.

[12] Drummond, O. E., Ogle, T. L., and Waugh, S., "Metrics for evaluating track covariance consistency," Proceedings of the SPIE, Vol. 6699, 2007.

[13] Tapley, B. D., Schutz, B. E., and Born, G. H., Statistical Orbit Determination, Elsevier Academic Press, Burlington, MA, USA, 2004.

[14] Jazwinski, A. H., Stochastic Processes and Filtering Theory, Vol. 64 of Mathematics in Science and Engineering, Academic Press, Inc., New York, NY 10003, USA, 1970.

[15] Zaidi, W. and Hejduk, M. D., "Earth Observing System Covariance Realism," AIAA/AAS Astrodynamics Specialist Conference, AIAA SPACE Forum, , No. AIAA 2016-5628.

[16] Gates, C., A Simplified Model of Midcourse Maneuver Execution Errors, JPL technical report, Jet Propulsion Laboratory, California Institute of Technology, 1963.

[17] Wagner, S. V. and Goodson, T. D., "Execution-error modeling and analysis of the Cassini-Huygens spacecraft through 2007," No. AAS 08-113.

[18] Wagner, S. V., Arrieta, J., Hahn, Y., Stumpf, P. W., Valerino, P. N., and Wong, M. C., "Cassini Solstice Mission maneuver experience: year three," No. AAS 13-717. 